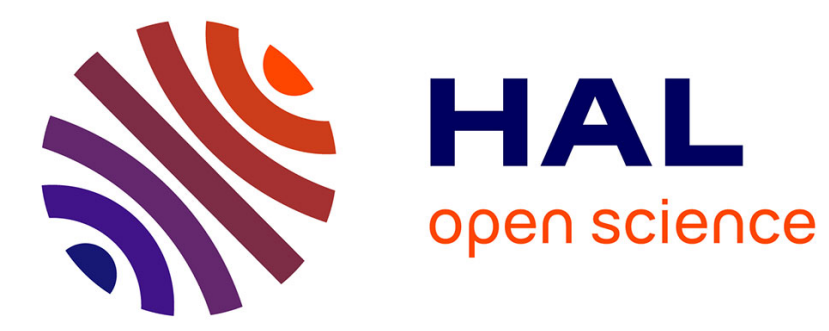

\title{
Bilateral teleoperation transparency at transient states
}

Pierre-Philippe Robet, Sylvain Devie, Yannick Aoustin, Maxime Gautier

\section{To cite this version:}

Pierre-Philippe Robet, Sylvain Devie, Yannick Aoustin, Maxime Gautier. Bilateral teleoperation transparency at transient states. 14th Conference on Control on Industry Applications (Induscon 2021), Aug 2021, Sao Paulo, Brazil. 10.1109/INDUSCON51756.2021.9529857 . hal-03414315

\section{HAL Id: hal-03414315 \\ https://hal.science/hal-03414315}

Submitted on 9 Nov 2021

HAL is a multi-disciplinary open access archive for the deposit and dissemination of scientific research documents, whether they are published or not. The documents may come from teaching and research institutions in France or abroad, or from public or private research centers.
L'archive ouverte pluridisciplinaire HAL, est destinée au dépôt et à la diffusion de documents scientifiques de niveau recherche, publiés ou non, émanant des établissements d'enseignement et de recherche français ou étrangers, des laboratoires publics ou privés. 


\section{Bilateral teleoperation transparency at transient states}

\author{
Pierre-philippe Robet \\ University of Nantes, LS2N, \\ 1, rue de la Noë - BP 92101 \\ 44321 Nantes Cedex 03 \\ Pierre-philippe.robet@1s2n.fr
}

\author{
Sylvain Devie \\ University of Nantes, LS2N, \\ 1, rue de la Noë - BP 92101 \\ 44321 Nantes Cedex 03 \\ Sylvain.Devie@1s2n.fr
}

\author{
Yannick Aoustin \\ University of Nantes, LS2N, \\ 1, rue de la Noë - BP 92101 \\ 44321 Nantes Cedex 03 \\ Yannick.aoustin@1s2n.fr
}

\author{
Maxime Gautier \\ University of Nantes, LS2N, \\ 1 , rue de la Noë - BP 92101 \\ 44321 Nantes Cedex 03 \\ Maxime.gautier@1s2n.fr
}

\begin{abstract}
To perform an epidural, a needle is inserted through the patient's skin and guided between the patient's vertebrae to the yellow ligament, which is resistant. Once the yellow ligament has passed, the needle must stop so that the anesthetic fluid in the epidural space can be injected. What makes this operation complicated is that the resistance of the yellow ligament varies greatly depending on the patient's physiognomy. To enhance the sensations of the anesthetist, we proposed to use a bilateral teleoperation system. The anesthetist manipulates a master arm while a needle is fixed on the slave arm to perform the epidural operation. In this paper, we discuss the tuning of the gains to reach ideal transparency at steady and transient states. Transparency depending on the number of control channels is calculated with the Hannaford matrix. Building on previous works, a new modeling, which allows extremely simple equations, is presented and a new experimental test-bed is proposed.
\end{abstract}

\section{Keywords-Teleoperation, Transparency, Mechatronic}

\section{INTRODUCTION}

Realizing the ideal bilateral teleoperation system is practically not an easy task. The ideal conditions require an exact knowledge of the master and slave dynamics and an exact measurement of speed and force signals. In addition to obtain ideal transparency at steady and transient states the control structure and the control law has to be taken into account.

Ohnishi et al. [1] gives a good overview of the states of the art about the teleoperated systems during the nineties. Two main approaches can be cited: the Hannaford et al. one [2], the Lawrence et al. [3]. Both approach introduced matrix where the velocity of the master robot and the velocity of the slave robot are combined into equations with forces exerted on each robot. Then a passivity criterion is defined to study the ideal transparency of the teleoperation system.

One of the main issues of a teleoperated system is the time delay for the information transmission between two robots [4], [5], [6]. The sampling effect is analysed in [7]. In [8] the transmission delay is supposed to be unknown and nonconstant. Latency issue with different types of wireless communication methods is study in [9]. In this paper, the electronic connections, the proximity of the two robots and the use of a single piece of hardware for the control part allow us to neglect this delay.

Industrial robots have important friction and damping coefficients [10]. Depending on the number of channels of the teleoperation system, this disadvantage can be canceled to obtain ideal transparency at steady states [11].

In [12] [13], the authors discuss the best place to integrate the force sensor between the base of the robot and its endeffector. According to this study, a force sensor placed at the end-effector of the robot, allows a better rejection of the inner frictions. Placing the sensor at the base allows to measure an environment effort at any point of the robot. In this paper the force sensor are at the end-effector of each one degree of freedom (dof) prismatic robots [14].

As it is shown in [15] [16] natural transparency of the fourchannel is possible at steady states. This is not the case with the two-channel position to position $(P P)$ or the force to position $(F P)$ or the position to force $(P F)$ teleoperation architecture. But in [17] [18], it is shown under particular conditions that transparency at steady states can be achieved with a two-channel force to force $(F F)$ teleoperation. Even with the four-channel, the ideal transparency at transient states is difficult to realised, but can be enhanced [19].

We have defined a system that allows the controller to be tuned using frequency analysis to achieve the desired performance of each robot. The position and strength of each robot depend on the environment and the interaction between each robot [20]. Transparency conditions at steady states and transient states are studied. To carry out this study a modeling which allows extremely simple equations compared to [11] and [21] is used. Consequently, it becomes very easy to get the analytical results to check the transparency at steady states and transient states; this will be the main contribution of this paper.

This paper is outlined as follows: Section II describes the modeling of a robot- $i$. Section III presents our controllers and the four-channel bilateral teleoperation. Section IV deals with the Hannaford formalist applied to the four and the twochannel bilateral teleoperation. Section $\mathrm{V}$ focuses on the ideal steady states transparency and to the ideal transient states transparency which brings more constraints.

\section{MODELING}

Two EMPS (Electro Mechanical Positioning System) robots are considered Fig. 1. The inner flexibility of the robot can be neglected when compared to that of the force sensor. All variables are given in SI units on the load side. Let us define $F_{m i}=G_{\tau i} I_{r e f i}$, the force from de motor applied on the load side of the robot- $i$, where $I_{\text {ref } i}$ is the reference current and $G_{\tau i}(N / A)$ the force constant.

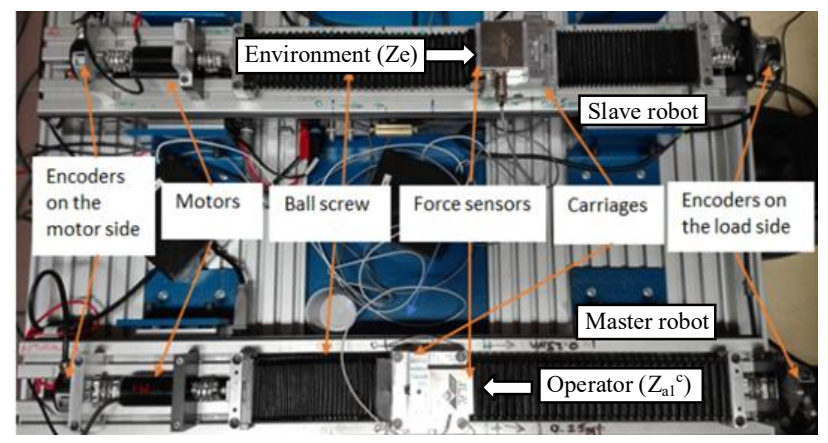

Fig. 1 EMPS robot main components 
This sensor is a spring with a stiffness coefficient $K_{r i}$, which is given by the manufacturer and has been checked experimentally. On Fig. 2, the position $\delta_{i}$ is the relative deformation of the force sensor spring defined in order to have $F_{i}=0$ when $\delta_{i}=0$. The considered mechanical system has two dofs : a rigid dof $q_{i}$ and a flexible $\operatorname{dof} \delta_{i}$.

The position $q_{i}$ of the end effector of the robot and the position $x_{i}$ of the end-effector are such that: $x_{i}=q_{i}+\delta_{i}$

$M_{i}$ is the mass of carriage and the equivalent mass in translation of the motor and the screw inertia $(\mathrm{kg}), f_{v i}$ is the viscous damping coefficient $(\mathrm{N} /(\mathrm{m} / \mathrm{s}))$ and $f_{c i}$ is the Coulomb friction $(N) . m_{i}$ is the mass $(\mathrm{kg})$ of the force sensor and the tool. The friction forces on this body are neglected in front of the external forces.

With respect to the reference frame fixed to the robot, the dynamic model of the horizontal mechanical device is:

$$
\begin{aligned}
& F_{i}^{*}=m_{i} \ddot{x}_{i}+F_{i} \quad \text { and } F_{i}=K_{r i}\left(x_{i}-q_{i}\right) \\
& F_{i}+F_{m i}=M_{i} \ddot{q}_{i}+f_{v i} \dot{q}_{i}+f_{c i} \operatorname{sign}\left(\dot{q}_{i}\right)
\end{aligned}
$$

with $F_{m i}$ the actuation force on carriage mass $M_{i}$ and $F_{i}^{*}$ the interaction force from the environment on mass $m_{i}$.

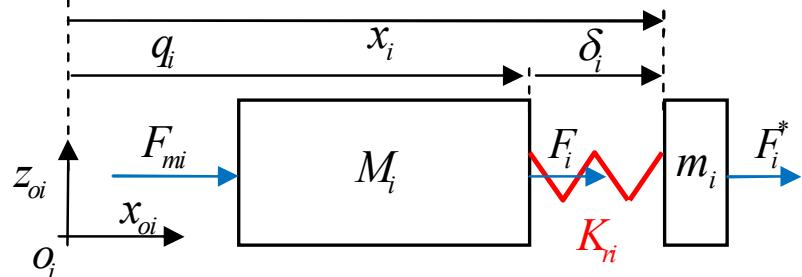

Fig. 2 Scheme of the moving part of the robot, the force sensor and tool

In the following, let us differentiate the force $F_{i}^{*}$ applied on the system by the environment and the force $F_{i}=K_{r i}\left(x_{i}-q_{i}\right)$ measured by the force sensor. The interaction force $F_{i}^{*}$ applied on the system and the velocity $\dot{x}_{i}$ are linked by the impedance of the environment: $Z_{e i}=\frac{F_{i}^{*}}{\dot{x}_{i}}$. This environment consists of the actions of the operator for the master robot and the reactions of the obstacle for the slave robot.

\section{Control Design}

\section{A. Focus on a single robot}

In classical applications, we consider a light tool and the mass $m_{i}$ is neglected. The Coulomb friction $f_{c i}$ is assimilated to a constant perturbation, corrected by the integral action of the inner loop. Thus the robot- $i$ model can be described by Fig. 3 .

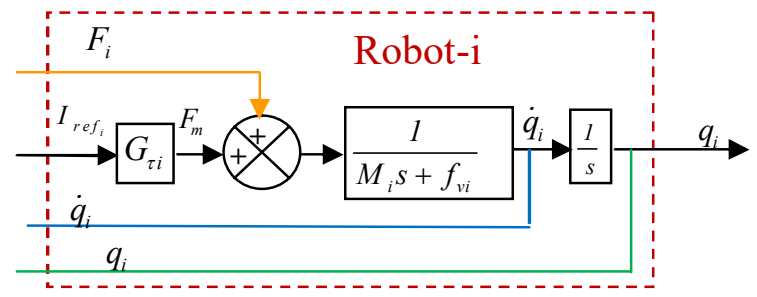

Fig. 3 Model of the robot- $i$
The considered controller is presented in Fig. 4 for robot- $i$ and uses an inner IP velocity loop allowing us to tune the dynamic behaviour of the robot. The aim of these outer loops is to tune the apparent impedance of the robot around an equilibrium position.

Because, the frequency range of the force done by the human hands is ten times smaller than the bandwidth of the velocity loop, around an equilibrium point $F_{i}^{*} \simeq F_{i}=-K_{r i} q_{i}$

- The apparent impedance $Z_{a i}$, calculated around an equilibrium position leads us to choose: $F_{\text {iref }}=0$ and $q_{\text {iref }}=0$. For the robot $-i$, it comes:

$Z_{a i}=\frac{F_{i}}{\dot{q}_{i}}=\left(M_{i} s+\left(f_{v i}+K_{v i} t_{v i}\right)+\frac{K_{v i}}{s}+\frac{K_{v i}}{s^{2}} k_{p i}\right)\left(1+\frac{k_{e i} K_{v i}}{s}\right)^{-1}$

With $Z_{a i}=M_{a i} s+B_{a i}+\frac{K_{a i}}{s}$, we have $M_{a i}$ the apparent mass, $B_{a i}$ the apparent viscous friction coefficient and $K_{a i}$ the apparent stiffness. According to the definition of these coefficients, the outer force loop and the outer position loop can be used to tune the apparent stiffness and the apparent viscous fiction coefficient of the system.

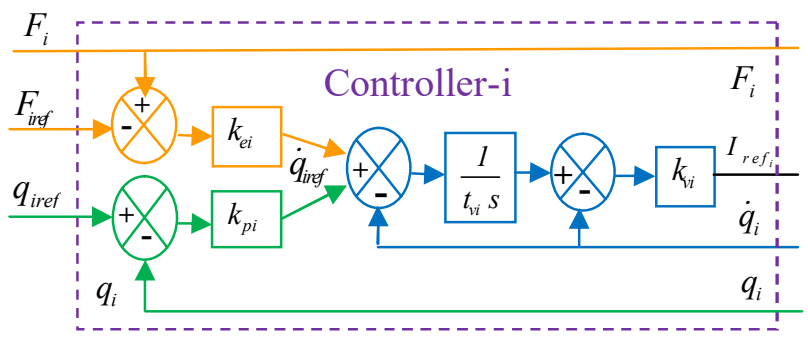

Fig. 4 Controller of speed, position and force of robot- $i$

With the approximation in papers [21][11] to get the master apparent impedance $Z_{a l}^{c}$ of the coupled system, we have done the following approximation: $1<<\frac{k_{i i} K_{v i}}{s}, f_{v i}<<K_{v i} t_{v i}$ ,$M_{i} s^{2} \ll<K_{v i} t_{v i} s$, so that:

$$
Z_{a i}=M_{a i} s+B_{a i}+\frac{K_{a i}}{s} \simeq \frac{t_{v i}}{k_{e i}} s+\frac{1}{k_{e i}}+\frac{k_{p i}}{k_{e i} s}
$$

With the new approximation done in this paper: $1<<\frac{k_{e i} K_{v i}}{s}$, $M_{i} s^{2}<<K_{v i} t_{v i} s$, it gives a more complicated expression of $Z_{a i}$ used into the calculation of the bilateral teleoperation.

$$
Z_{a i}=M_{a i} s+B_{a i}+\frac{K_{a i}}{s} \simeq\left(\frac{f_{v i}+K_{v i} t_{v i}}{K_{v i} k_{e i}}\right) s+\frac{1}{k_{e i}}+\frac{k_{p i}}{k_{e i} s}
$$

But during the demonstration to obtain equation (4), it appears a simplification allowing very simple expression at the end. In a Bode point of view, neglecting or not $f_{v i}<<K_{v i} t_{v i}$ gives the same Bode plot in the working frequency base band. Nevertheless neglecting $1<<\frac{k_{e i} K_{v i}}{s}$ and $M_{i} s^{2}<<K_{v i} t_{v i} s$ gives two different Bode plot of $1 / Z_{a i}$ as shown on Fig. 10. The difference appears at a frequency greater that $50 \mathrm{hz}$ and starts to be significant for a frequency of $80 \mathrm{~Hz}$. But, the movement of a human hands and fingers has a maximum frequency of $8 \mathrm{~Hz}[22]$. Thus the proposed 
approximation of $Z_{a i}$ transfer function is similar to the real one into teleoperation base band.

\section{B. Four-channel bilateral teleoperation}

Between the two robots there is a four-channel bilateral coupling, see Fig. 5. Each robot- $i$ includes the controller and the mechanism as in Fig. 3 and Fig. 4. Let us put $Z_{e}$ the impedance of the environment. The interaction between the robot and the environment is performed via a force sensor having a stiffness $K_{r 2}$. In this condition, the maximal stiffness of the group \{environment + sensor \} is $K_{r 2}$.

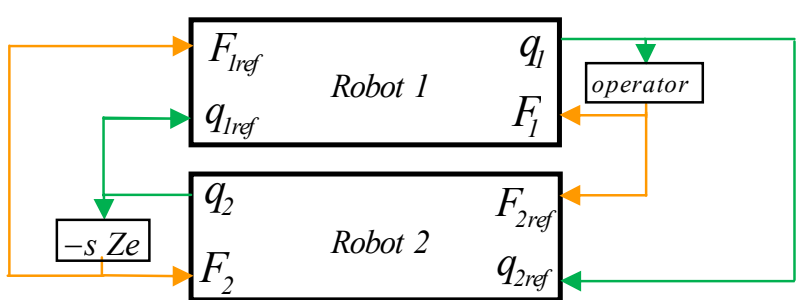

Fig. 5 Four-channel bilateral teleoperation system

The operator feels impedance $Z_{a l}^{c}=\left.\frac{F_{1}}{\dot{q}_{1}}\right|_{F_{2}=-Z_{e} \dot{q}_{2}}$ for the coupled system, which depends on the apparent impedance of each robot and how the system is coupled.

This impedance of the coupled system has to be calculated in order to tune the transparency of the system. Based on Fig. 3, Fig. 4, Fig. 5 the linear control system of the two robots, and taking into account the approximation we used for the calculation of $Z_{a i}$ gives :

$$
F_{i}-F_{\text {iref }}=Z_{a 1} \dot{q}_{i}-\dot{q}_{\text {iref }} K_{a i} / s
$$

For the four bilateral teleoperation with $F_{\text {lref }}=-F_{2}$, $F_{2 r e f}=-F_{1}$ and $\dot{q}_{1 r e f}=\dot{q}_{2}, \dot{q}_{2 r e f}=\dot{q}_{1}$, it comes:

$$
\begin{aligned}
& F_{1}+F_{2}=Z_{a 1} \dot{q}_{1}-\dot{q}_{2} K_{a 1} / s \\
& F_{2}+F_{1}=Z_{a 2} \dot{q}_{2}-\dot{q}_{1} K_{a 2} / s
\end{aligned}
$$

\section{HANNAFORD MATRIX APPLIED TO OUR MODEL}

The four-channel bilateral teleoperation given by Fig. 5 can be study with the modified Hannaford representation given on Fig. 6.

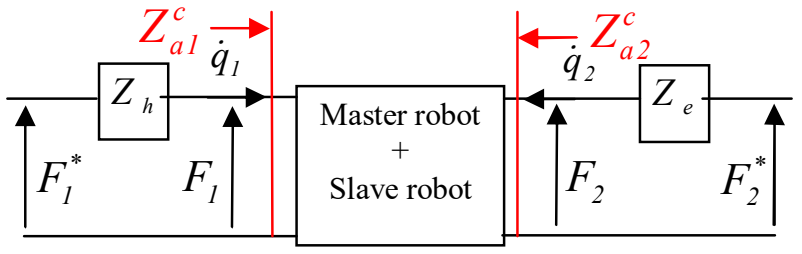

Fig. 6 Modified Hannaford representation for teleoperation

In the Hannaford [2] representation, $\dot{q}_{2}$ has an opposite sign. The proposed representation has the advantage to get more symmetrical equation without changing anything. Calculation can be done easily by changing the indices $i, j=1,2$ and $Z_{h} \leftrightarrow Z_{e}$ to deduce results without calculation when apparent impedance $Z_{a i}^{c}$ calculation has to be done.

This representation gives the following equations:

$$
\begin{aligned}
& F_{1}=F_{1}^{*}-Z_{h} \dot{q}_{1} \\
& F_{2}=F_{2}^{*}-Z_{e} \dot{q}_{2}
\end{aligned}
$$

where impedances $Z_{h}$ and $Z_{e}$ are linked with the dynamics of the master and slave robots when the operator and the environment are in contact.

The master-slave system can be represented by two-port network models such as the hybrid model :

$$
\left[\begin{array}{l}
F_{1} \\
\dot{q}_{2}
\end{array}\right]=\left[\begin{array}{ll}
h_{11} & h_{12} \\
h_{21} & h_{22}
\end{array}\right]\left[\begin{array}{l}
\dot{q}_{1} \\
F_{2}
\end{array}\right]
$$

where the hybrid parameters $h_{i j}$ are functions of the master and slave dynamics and their control parameters.

The apparent impedance of the master is defined as:

$$
Z_{a 1}^{c}=\left.\frac{F_{1}}{\dot{q}_{1}}\right|_{F_{2}^{*}=0}=\frac{h_{11}+\left(h_{11} h_{22}-h_{12} h_{21}\right) Z_{e}}{1+h_{22} Z_{e}}
$$

With our modified representation, the Hannaford criterion [2] of transparency is not modified and given by :

$$
h_{12} h_{21}=-1, h_{22}=0, h_{11}=0
$$

So that the operator, at steady states and transient states, feels impedance : $\quad Z_{a 1}^{c}=\left.\frac{F_{1}}{\dot{q}_{1}}\right|_{F_{2}^{*}=0}=Z_{e}$

\section{A. Four-channel bilateral teleoperation with Hannaford}

Hannaford hybrid parameters are obtained with (5), (6), (7)

$$
\begin{aligned}
& h_{11}=\frac{Z_{a 1} Z_{a 2}-K_{a 1} K_{a 2} / s^{2}}{Z_{a 2}+K_{a 1} / s}, \quad h_{12}=-1 \\
& h_{21}=\frac{Z_{a 1}+K_{a 2} / s}{Z_{a 2}+K_{a 1} / s}, \quad h_{22}=0
\end{aligned}
$$

The apparent impedance of the robot-1-2 is given by:

$$
Z_{a 1}=M_{a 1} s+B_{a 1}+\frac{K_{a 1}}{s}, \quad Z_{a 2}=M_{a 2} s+B_{a 2}+\frac{K_{a 2}}{s}
$$

Equation (9) is checked if $h_{12} h_{21}=-1 \Leftrightarrow h_{2 l}=1$, consequently, at low frequency $h_{21} \underset{s \rightarrow 0}{\approx} \frac{K_{a 1}+K_{a 2}}{K_{a 1}+K_{a 2}}=1$ and $h_{11} \underset{s \rightarrow 0}{\approx} \frac{B_{a 1} K_{a 2}+B_{a 2} K_{a 1}}{K_{a 2}+K_{a 1}} \underset{B_{a 1}=B_{a 2}}{=} B_{a i} \underset{k_{e i} \rightarrow \infty}{\rightarrow}$ or $\underset{K_{a 1}=K_{a 2}}{=} \frac{B_{a 1}+B_{a 2}}{2} \underset{k_{e i} \rightarrow \infty}{\rightarrow} 0$ Including transient states, the ideal transparency depends on the apparent viscous friction coefficients $B_{a 1}, B_{a_{2}}$ of the slave robot and the master robot.

\section{B. Two-channel bilateral teleoperation with Hannaford}

Based on the previous study, a two-channel bilateral teleoperation system can be defined by limiting the interaction between the two robots. In the preceding section, each robot is controlled according to the position of the other robot as well as the force it exchanges with its environment. However, here each robot will be controlled with only one of these channels of information.

a) Force to Force (FF)

If the robot- $i$ is controlled thanks to the effort of the other robot, the force reference $F_{\text {iref }}$ will be defined, but not the position reference $q_{i r e f}$. It implies that in (3) $k_{p i}=0$, the 
direct consequence is $K_{a i}=0$ into (4) and (3). Hannaford hybrid parameters are obtained with(5), (6), (7) :

$h_{11}=Z_{a 1}$ et $h_{12}=-1 ; h_{21}=\frac{Z_{a 1}}{Z_{a 2}}$ et $h_{22}=0$

With the two-channel (FF), $\quad Z_{a l}=M_{a l} s+B_{a l}$, $Z_{a 2}=M_{a 2} s+B_{a 2}$, thus equation (9) is checked if $h_{12} h_{21}=-1 \Leftrightarrow \frac{Z_{a 1}}{Z_{a 2}}=1$, consequently, at low frequency $h_{21} \underset{s \rightarrow 0}{\approx} \frac{B_{a 1}}{B_{a 2}}=1$ and $h_{11} \underset{s \rightarrow 0}{\approx} B_{a i} \underset{B_{a i} \rightarrow \infty}{\rightarrow} 0$

The ideal transparency, including transient states, depends on the apparent viscous friction coefficients $B_{a 1}, B_{a 2}$ of the slave robot and the master robot.

\section{b) Position to Position (PP)}

If the robot- $i$ is controlled thanks to the position of the other robot, the position reference $q_{\text {iref }}$ will be defined, but not the force reference, which will be set to zero into (4). Hannaford hybrid parameters are obtained with (5), (6), (7):

$$
\begin{aligned}
& h_{11}=\frac{Z_{a 1} Z_{a 2}-K_{a 1} K_{a 2} / s^{2}}{Z_{a 2}}, h_{12}=-\frac{K_{a 1} / s}{Z_{a 2}} \\
& h_{21}=\frac{K_{a 2} / s}{Z_{a 2}}, \quad h_{22}=\frac{1}{Z_{a 2}}
\end{aligned}
$$

With the two-channel $(P P), \quad Z_{a l}=M_{a l} s+B_{a l}+\frac{K_{a 1}}{s}$, $Z_{a 2}=M_{a 2} s+B_{a 2}+\frac{K_{a 2}}{s}$, thus equation (9) is checked if $h_{12} h_{21}=-1 \Leftrightarrow \frac{-K_{a 1} K_{a 2}}{s^{2} Z_{a 2}{ }^{2}}=-1, \quad$ consequently, at low frequency: $h_{12} h_{21} \underset{s \rightarrow 0}{\approx}-\frac{K_{a 1}}{K_{a 2}} \underset{K_{a 1}=K_{a 2}}{=}$

$$
\text { and } \begin{aligned}
h_{11} \underset{s \rightarrow 0}{\approx} \frac{B_{a 1} K_{a 2}+B_{a 2} K_{a 1}}{K_{a 2}} \underset{K_{a 1}=K_{a 2}}{=} B_{a 1}+B_{a 2} \underset{k_{e i} \rightarrow \infty}{\rightarrow 0} \\
h_{11} \underset{s \rightarrow 0}{\approx} \frac{B_{a 1} K_{a 2}+B_{a 2} K_{a 1}}{K_{a 2}} \underset{\substack{B_{a 1}=B_{a 2} \\
=}}{=} B_{a i}\left(1+\frac{K_{a 1}}{K_{a 2}}\right) \underset{\substack{k_{e i} \rightarrow \infty \\
k_{p 2} \geq k_{p 1}}}{\rightarrow 0}
\end{aligned}
$$

and $h_{22} \underset{s \rightarrow 0}{\approx} \frac{s}{K_{a 2}} \underset{\substack{k_{p 2} / k_{e 2} \rightarrow \infty \\ k_{p 2}>k_{e 2}}}{\rightarrow} \underset{0}{0}$

The transparency, including transient states, depends on the apparent viscous friction coefficients $B_{a 1}, B_{a 2}$ and the apparent stiffness coefficients $K_{a 1}, K_{a 2}$ of both robots. This is the worst case. Four parameters have to be managed with the position to position $(P P)$ two-channel control law to go toward good transparency at transient states.

\section{- Experimental result of the two-channel (PP)}

These robots were identified for this study in [23]. The parameters are: $M_{1}=105 \mathrm{~kg}, f_{v 1}=313 \mathrm{~N} /(\mathrm{m} / \mathrm{s}), f_{c 1}=13 \mathrm{~N}$, $G_{\tau 1}=12 \mathrm{~N} / \mathrm{A}$ and $K_{r 1}=210^{4} \mathrm{~N} / \mathrm{m}$ for the master robot and $M_{2}=102 \mathrm{~kg}, f_{v 2}=340 \mathrm{~N} /(\mathrm{m} / \mathrm{s}), f_{c 2}=18 \mathrm{~N}, G_{\tau 2}=12 \mathrm{~N} / \mathrm{A}$ and $K_{r 2}=410^{4} \mathrm{~N} / \mathrm{m}$ for the slave robot. Both robots are controlled thanks to an input/output DSpace card. A single system is controlling both robots, which are located in the same small area, minimizing the time delay. The experiments are performed with the following protocol: the operator applies a specific effort on robot 1 while maintaining contact. The robot 2 is in contact with a stiff environment. This passive environment can push the robot when it is in contact, but not pull it. Two external force sensors are placed on the end-effector of the robot, measuring the efforts of the operator and the stiff environment. And there is no problem of discretization because the sampling rate of the system is 100 times higher than the working frequency. The new experimental tested bed includes analyses of bode of the Hannaford parameters.

Before $5 \mathrm{~s}$, the robot 2 is not in contact with the environment thus the force $F_{2}$ is equal to $0 N$ while a force $F_{1}$ is variable. Carriages of both robots are moving and the $(P P)$ control law gives a good tracking of velocity and position. After $4 \mathrm{~s}$, the robot 2 is in contact with the environment and the tracking of force is good. But for the velocity and position tracking it appears ripples due to the virtual spring effect. To decrease the tracking error of velocity and position, $k_{p 1}, k_{e l}$ has to be tuned using (13), (14) and (15).
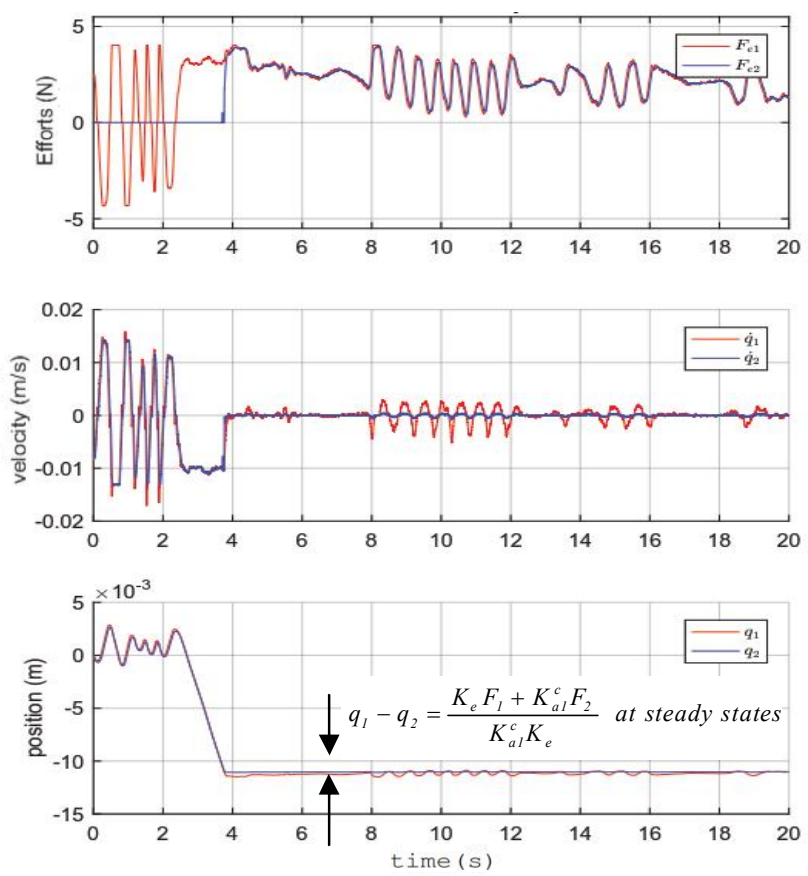

Fig. 7 Two-channel bilateral teleoperation $(P P)$

Let us have a look to the Hannaford condition of transparency, including transient states, gives by (13),(14) ,(15). For the experimental study of the two-channel $(P P)$, the apparent viscous value is : $B_{a i}=1 / k_{e i}=444 \rightarrow 53 \mathrm{~dB}$. We choose $k_{p i}>>k_{e i}$, so that the apparent stiffness value is : $K_{a i}=k_{p i} / k_{e i}=20000 \rightarrow 86 \mathrm{~dB}$. Let us recall that the working frequency range of the movement of a human hands and fingers has a maximum frequency of $8 \mathrm{~Hz}$ [24].

The bode plot of $h_{12} h_{21}$ on Fig. 8 show that, at low frequency, the magnitude go toward zero and the phase margin go to $180^{\circ}$. Thus equation (13) is verified for teleoperation frequency range. 


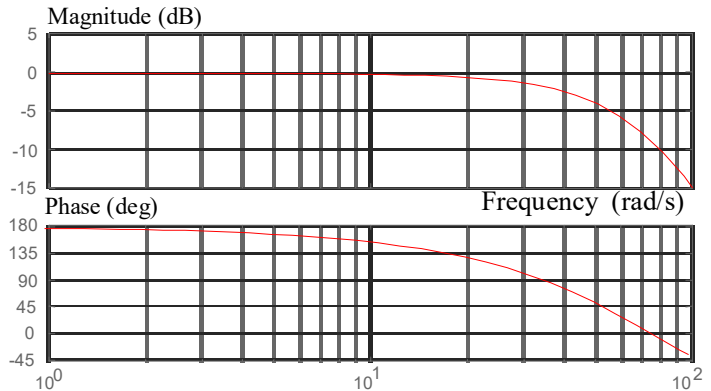

Fig. $8 \mathrm{~h}_{12} \mathrm{~h}_{21}$ Bode diagram (PP)

As shown on Fig. 9, the bode plot of $h_{1 I}$ at low frequency gives a magnitude of $2 B_{a i}=888 \rightarrow 59 \mathrm{~dB}$ and a phase margin of zero. Consequently (14) is not validated at transient states. The solution is to increase $k_{p i}>>k_{e i}$, but the teleoperation system become instable. This result shows the compromise between stability and transient transparency.

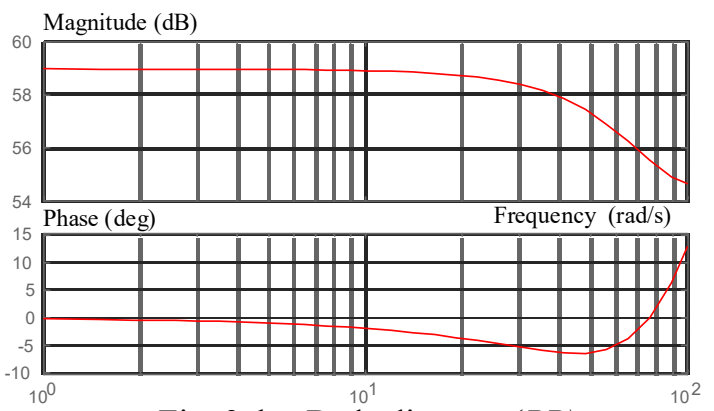

Fig. $9 \mathrm{~h}_{11}$ Bode diagram $(P P)$

The last condition of transparency is given by (15). Depending on $K_{a i}=k_{p i} / k_{e i}=86 \mathrm{~dB}$, the $h_{22}$ magnitude goes toward zero as shown on Fig. 10. It value is around $-86 \mathrm{~dB}$ at a frequency of $1 \mathrm{rad} / \mathrm{s}$.

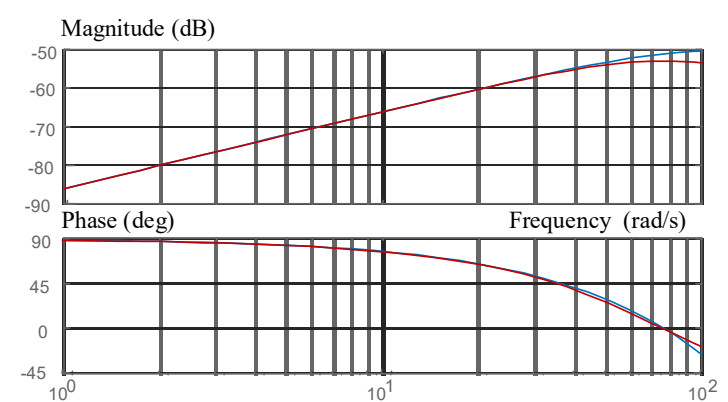

Fig. $10 \quad \mathrm{~h}_{22}=1 / \mathrm{Z}_{\mathrm{ai}}$ Bode diagram $(P P)$

c) Force to Position (FP)

In that hybrid case $F P$, the master is controlled thanks to the effort, so the force reference $F_{\text {lref }}$ is defined, but $F_{2 \text { ref }}=0$. The slave robot is controlled in position, so the position reference $q_{2 \text { ref }}$ is defined, but $q_{1 \text { ref }}=0$. It implies that $k_{p 1}=0$ into (3), the direct consequence is $K_{a l}=0$ into (4) and (3).

Hannaford hybrid parameters are obtained with(5), (6), (7) :

$$
h_{11}=Z_{a 1}, \quad h_{12}=-1, \quad h_{21}=\frac{K_{a 2} / s}{Z_{a 2}}, \quad h_{22}=\frac{1}{Z_{a 2}}
$$

With the two-channel (FP), $\quad Z_{a l}=M_{a 1} s+B_{a l}$, $Z_{a 2}=M_{a 2} s+B_{a 2}+\frac{K_{a 2}}{s}$, thus equation (9) is checked if $h_{12} h_{21}=-1 \Leftrightarrow \frac{-K_{a 2}}{s Z_{a 2}}=-1$. Consequently, at low frequency we have: $h_{12} h_{21} \underset{s \rightarrow 0}{\approx}-\frac{K_{a 2}}{K_{a 2}}=-1$ and $h_{22} \underset{\substack{\approx \rightarrow 0 \\ K_{a 2}}}{\approx} \underset{K_{a 2}}{\rightarrow} \underset{\substack{k_{p 2} / k_{c 2} \rightarrow \infty \\ k_{p 2}>k_{e 2}}}{0}$. We also have the following result : $h_{11} \underset{s \rightarrow 0}{\approx} B_{a l}=\frac{1}{k_{e l}} \underset{k_{e l} \rightarrow \infty}{\rightarrow \rightarrow 0}$

The ideal transparency, including transient states, depends on the apparent viscous friction coefficient $B_{a l}$ of the master robot. It also depends on $B_{a 2}=1 / k_{e 2}$ the apparent viscous friction coefficient of the slave robot, due to the dependence on $K_{a 2}=k_{p 2} / k_{e 2}$ the apparent stiffness of the slave robot.

\section{d) Position to Force (PF)}

In that hybrid case $P F$, the master is controlled thanks to the position, so the position reference $q_{\text {lref }}$ is defined, but $q_{2 \text { ref }}=0$. The slave robot is controlled in force, so the reference is defined, but $F_{1 r e f}=0$. It implies that $k_{p_{2}}=0$ into (3), the direct consequence is $K_{a 2}=0$ into (4) and (3). Hannaford hybrid parameters are obtained with(5), (6), (7) :

$$
\begin{aligned}
& h_{11}=\frac{Z_{a 1} Z_{a 2}}{Z_{a 2}+K_{a 1} / s}, \quad h_{12}=-\frac{K_{a 1} / s}{Z_{a 2}+K_{a 1} / s} \\
& h_{21}=\frac{Z_{a 1}}{Z_{a 2}+K_{a 1} / s}, \quad h_{22}=\frac{1}{Z_{a 2}+K_{a 1} / s}
\end{aligned}
$$

With the two-channel $(P F), Z_{a 1}=M_{a l} s+B_{a 1}+\frac{K_{a 1}}{s}$,

$Z_{a 2}=M_{a 2} s+B_{a 2}$ thus equation (9) is checked if:

$h_{12} h_{21}=-1 \Leftrightarrow \frac{-K_{a 1} Z_{a 1}}{s\left(Z_{a 2}+K_{a 1} / s\right)^{2}}=-1$, consequently, at low frequency $h_{12} h_{21} \underset{s \rightarrow 0}{\approx}-\frac{K_{a 1}{ }^{2}}{K_{a 1}{ }^{2}}=-1$ and $h_{22} \underset{s \rightarrow 0}{\approx} \frac{s}{K_{a 1}} \underset{\substack{k_{p l} / k_{e l} \rightarrow \infty \\ k_{p l}>k_{e l}}}{\rightarrow 0}$ and also we have $h_{11} \underset{s \rightarrow 0}{\approx} B_{a 2}=\frac{1}{k_{e 2}} \underset{k_{e 2} \rightarrow \infty}{\rightarrow} 0$.

The ideal transparency, including transient states, depends on the apparent viscous friction coefficients $B_{a 2}$ of the slave robot. It also depends on the apparent stiffness coefficient $K_{a 1}=k_{p l} / k_{e l}$ of the master robot. Consequently, it depends on $B_{a 1}=1 / k_{e l}$ of the master robot.

\section{STEADY STATES AND TRANSIENT STATES}

Considering the steady states, the apparent impedance $Z_{a l}^{c}$ can be calculated with $Z_{e}=K_{e} / s$, where $K_{e}$ is the stiffness of the sensor+environment. The steady states transparency is given when the speed of both robots is zero. The apparent stiffness $K_{a l}^{c}$ felt by the operator at steady states is defined as: $K_{a l}^{c}=\lim _{s \rightarrow 0} s Z_{a l}^{c}$ and can be calculated with (8) and gives the same results that those obtained in [11]. 


$$
\begin{aligned}
& K_{a 1}^{c} \underset{4 \text { channels }}{=} K_{e}, \quad K_{a 1}^{c} \underset{\text { 2channels (FF) }}{=}\left(B_{a 1} / B_{a 2}\right) K_{e} \underset{B_{a 1}=B_{a 2}}{=} K_{e} \\
& K_{a 1}^{c} \underset{\text { 2channels (PP) }}{=}\left(K_{a 1} K_{e}\right) /\left(K_{a 2}+K_{e}\right) \underset{K_{a 1}=K_{a 2}>>K_{e}}{\rightarrow} K_{e} \\
& K_{a 1}^{c} \underset{\text { 2channels (FP) }}{=}\left(K_{a 2} K_{e}\right) /\left(K_{a 2}+K_{e}\right) \underset{K_{a 2} \gg K_{e}}{\rightarrow} K_{e} \\
& K_{a 1}^{c} \underset{\text { 2channels (PF) }}{=}\left(K_{a 1} K_{e}\right) /\left(K_{a 1}+K_{e}\right) \underset{K_{a l} \gg K_{e}}{\rightarrow} K_{e}
\end{aligned}
$$

It appears that $h_{12} h_{21}=-1, h_{22}=0$ Hannaford criterions of transparency are related to steady states transparency ( $\dot{q}_{1}=\dot{q}_{2}=0$ ) comparing with equations (18). The perfect transparency at steady states is naturally obtained with the four-channel and possible with the two-channel $(F F)$ if $B_{a l}=B_{a 2}$. With the two-channel $(P P),(F P),(P F)$, it is only possible to go toward a perfect transparency at steady states by increasing $K_{a i}>>K_{e}$ and $K_{a i} \rightarrow \infty$.

At transient states, we need to add the $h_{11}=0$ parameter into the Hannaford criterions of ideal transparency. In that case, it appears that perfect transparency is not possible for the four-channel and the two-channel teleoperation. Assuming a compromise with stability, at low frequency with the four-channel and the two-channel $(F F)$ teleoperation system, it is possible to go toward transparency by decreasing $B_{a i} \rightarrow 0$. With the two-channel $(P P),(F P)$, $(P F)$, at transient states, to reach transparency, at low frequency it is necessary to decrease $B_{a i} \underset{k_{e i} \rightarrow \infty}{\rightarrow}$ and at the same time to increase $K_{a i} \gg K_{e}$ and $K_{a i} \rightarrow \infty$, which brings more constraints.

\section{CONCLUSION}

A haptic teleoperation system is presented. The originality of the tuning is done through closed loop of velocity, position and force. The resulting equation (3) of the dynamic gives coefficient that can be seen as the apparent mass $M_{a i}$, the apparent viscous friction coefficient $B_{a i}$ and the apparent stiffness $K_{a i}$. The transparency, including transient states, of the teleoperation system is studied with the Hannaford criteria. The apparent viscous friction coefficient is the main obstacle to get a perfect transparency at transient states. While it is possible to have perfect transparency at steady states with the four-channel and the two-channel $(F F)$, this is not the case with the two-channel $(F P),(P F),(P P)$. With the two-channel $(F P)$, the coefficient of apparent stiffness of the slave robot must be managed. It is that of the master with the two-channel $(P F)$. Because the two apparent stiffnesses must be adjusted at the same time with the two-channel $(P P)$, it is more difficult to obtain perfect transparency at steady states.

\section{REFERENCES}

[1] K. Ohnishi, M. Shibata, and T. Murakami, "Motion control for advanced mechatronics", IEEE/ASME Trans. on Mechatronics, vol. 1 , no. 1, pp. 56-67, 1996.

[2] B. Hannaford, "A Design Framework for Teleoperators with Kinesthetic Feedback", IEEE Trans. on Robotics and Automation, vol. 5, no. 4, pp. 426-434, 1989.

[3] D. A. Lawrence, "Stability and Transparency in Bilateral Teleoperation", IEEE Trans. on Robotics and Automation, vol. 9, no. 5, pp. 624-637, 1993.
[4] R. J. Anderson, M. W. Spong. "Bilateral control of teleoperation with time delay," IEEE Trans. on Automation Control, Vol.34, No.5, 1989, pp. 494-501.

[5] K. Hashtrudi-Zaad and S. E. Salcudean, "Transparency in TimeDelayed Systems and the Effect of Local Force Feedback for Transpar-ent Teleoperation," IEEE Trans. on Robotics and Automation, vol. 18, no. 1, pp. 108-114, 2002.

[6] J. Guo, C. Liu and P. Poignet, "Stable and enhanced position-force tracking for bilateral teleoperation with time delay", 2015 European Control Conference (ECC), Linz, 2015, pp. 1980-1985

[7] T. Yang, Y. L. Fu and M. Tavakoi, "An analysis of sampling effect on bilateral teleoperation system transparency", 2015 34th Chinese Control Conference (CCC), Hangzhou, 2015, pp. 5896-5900.

[8] C. Zhang and H. Wang, "Task-Space Adaptive Control of Bilateral Teleoperators with Time-Varying Delay", 2020 39th Chinese Control Conference (CCC), Shenyang, China, 2020, pp. 4586-4591.

[9] T. Mizoguchi, G. Kokubun, N. Yamaguchi, T. Shimono and K. Ohnishi, "Experimental Study on Bilateral Control System Under Different Wireless Communication Methods", 2019 IEEE International Conference on Mechatronics (ICM), Ilmenau, Germany, 2019, pp. 31-36.

[10] Kenta Seki, Shota Fujihara, Makoto Iwasaki, "Improvement of Force Transmission Performance Considering Nonlinear Friction in Bilateral Control Systems", IFAC PapersOnLine 50-1, pp 1207112076, July 2017.

[11] S. Devie, P.Ph. Robet, Y. Aoustin, M. Gautier. "Bilateral MasterSlave operation based on cascaded loop structure in force-position and velocity", In 2020 7th International Conference on Control, Decision and Information Technologies (CoDIT), juin 2020, Prague, République tchèque.

[12] G. Piolain, F. Geffard, A. Coudray, P. Garrec, J. F. Thro, and Y. Perrot, "Dedicated and standard industrial robots used as forcefeedback telemaintenance remote devices at the AREVA recycling plant", Int. Conf. on Applied Robotics for the Power Industry, CARPI 2010, pp. 1-6, 2010.

[13] F. Geffard, C. Andriot, A. Micaelli, and G. Morel, "On the use of a base force/torque sensor in teleoperation", IEEE Int. Conf. on Robotics and Automation., vol. 3, no. April, pp. 2677-2683, 2000.

[14] S. Devie, P.-p. Robet, Y. Aoustin, and M. Gautier, "Impedance control using a cascaded loop force control", IEEE Robotics \& Automation letter, pp. 1-7, 2018.

[15] M. Tavakoli, A. Aziminejad, R. V. Patel and M. Moallem, "Enhanced Transparency in Haptics-Based Master-Slave Systems", 2007 American Control Conference, New York, NY, 2007, pp. 14551460 .

[16] Z. Chen, F. Huang, W. Sun and W. Song, "An Improved WaveVariable Based Four-Channel Control Design in Bilateral Teleoperation System for Time-Delay Compensation”, IEEE Access, vol. 6, pp. 12848-12857, 2018.

[17] E. Naerum and B. Hannaford, "Global transparency analysis of the Lawrence teleoperator architecture", 2009 IEEE International Conference on Robotics and Automation, Kobe, 2009, pp 4344-4349.

[18] B. Willaert, D. Reynaerts, H. van Brussel and E. B. V. Poorten, "Bilateral Teleoperation: Quantifying the Requirements for and Restrictions of Ideal Transparency”, in IEEE Transactions on Control Systems Technology, vol. 22, no. 1, pp. 387-395, Jan. 2014.

[19] Z. Chen, F. Huang, C. Yang and B. Yao, "Adaptive Fuzzy Backstepping Control for Stable Nonlinear Bilateral Teleoperation Manipulators With Enhanced Transparency Performance", in IEEE Transactions on Industrial Electronics, vol. 67, no. 1, pp. 746-756, Jan. 2020.

[20] S. Devie, P. P. Robet, Y. Aoustin, M. Gautier, and A. Jubien, "Accurate force control and co-manipulation control using hybrid external command", IFAC World Congress, pp. 2271-2276, 2017.

[21] S. Devie, P.Ph. Robet, Y. Aoustin, M. Gautier. "A cascaded loop structure in force and position to control a bilateral teleoperation robotic systemn", In 6th international conference on control, decision and information technologies, mai 2019, Paris, France.

[22] H. -J. Freund, "Time control of hand movements", Elsevier, Progress in Brain Research, Volume 64, 1986, Pages 287-294.

[23] P.Ph. Robet, M. Gautier. "Parameters identification of an electrical actuator with methods based on IDIM, DIDIM, IDIM2, DIDIM2", In European Power Electronics and Drives Journal ; éd. Taylor \& Francis, 2018, vol. 28, num. 3.

[24] H.-J. Freund, "Time control of hand movements", Elsevier, Progress in Brain Research, Volume 64, 1986, Pages 287-294. 